Images dans le monde ibérique et ibéricoaméricain

5 | 2012

Le visible et l'invisible dans le monde hispanique et hispano-américain

\title{
Le visible saturé du Tratado llamado leche de la fe del príncipe christiano de Luis de Maluenda (1545)
}

Enjeux et limites du discours imagé en matière de spiritualité

\section{Fabrice Quero}

\section{(Q) OpenEdition \\ Journals}

Édition électronique

URL : http://journals.openedition.org/agedor/1011

DOI : 10.4000/agedor.1011

ISSN : 2104-3353

Éditeur

Laboratoire LISAA

Référence électronique

Fabrice Quero, «Le visible saturé du Tratado llamado leche de la fe del príncipe christiano de Luis de Maluenda (1545) », L'Âge d'or [En ligne], 5 | 2012, mis en ligne le 01 mars 2012, consulté le 16 décembre 2019. URL : http://journals.openedition.org/agedor/1011; DOI : 10.4000/agedor.1011 
Fabrice QUERO

Université Paris-Est Marne-la-Vallée, LISAA/EMHIS

\title{
LE VISIBLE SATURÉ DU TRATADO LLAMADO LECHE DE LA FE DEL PRÍNCIPE CHRISTIANO DE LUIS DE MALUENDA (1545) : ENJEUX ET LIMITES DU DISCOURS IMAGÉ EN MATIÈRE DE SPIRITUALITÉ
}

\begin{abstract}
Résumé : Le Tratado llamado leche de la fe del principe christiano (1545) du franciscain Luis de Maluenda est un centon d'Écritures saintes et d'écrits théologiques et spirituels. Le texte dans son ensemble, et son très long prologue en particulier, regorge d'images verbales selon une tradition qui remonte aux Écritures. Leur rôle déborde celui d'illustration mentale d'une vérité invisible par un objet visible ou visualisable. Elles offrent, en premier lieu, un indispensable agrément pour la lecture. Il s'agit toujours, par ailleurs, de métaphores mortes, autrement dit de métaphores non originales que le discours de Maluenda s'ingénie à décoder jusqu'à arriver, sous les yeux du lecteur, à l'épuisement de tous les ressorts de l'analogie. Les images perdent ainsi toute capacité à stimuler son imagination et deviennent un instrument de contrôle de sa méditation. Associées à une étouffante promotion du magistère ecclésiastique, elles ont tout d'un remède à des hérésies qui, en ces années, font de la liberté et de l'autonomie du chrétien la condition de son épanouissement spirituel.
\end{abstract}

Mots-clés : Maluenda, Luis de - XVIe siècle - Espagne - spiritualité - ascétisme - images verbales - magistère de l'église

Resumen: El Tratado llamado leche de la fe del príncipe christiano (1545) del franciscano Luis de Maluenda es un centón de Escrituras santas y de escritos teológicos y espirituales. El texto en su conjunto, y su muy largo prólogo de manera particular, rebosan de imágenes verbales, conforme a una tradición que se remonta a las Sagradas Escrituras. Su papel supera el de ilustrar, mentalmente, una verdad invisible gracias a un objeto visible o posible de visualizar. Ofrecen, primero, un imprescindible recreo para amenizar la lectura. Por otra parte, se trata siempre de metáforas muertas, es decir de metáforas que no son originales y que el discurso de Maluenda se empeña en descodificar hasta llegar, bajo los ojos del lector, al agotamiento de todos los elementos de la analogía. Pierden así las imágenes toda capacidad para estimular su imaginación y se convierten en un instrumento de control de su meditación. Asociadas a una agobiante promoción del magisterio eclesiástico, se asemejan mucho a un remedio a unas herejías que, en aquellos años, hacen de la libertad y de la autonomía del cristiano la condición de su realización espiritual.

Palabras clave: Maluenda, Luis de - siglo XVI - España - espiritualidad - ascetismo - imágenes verbales - magisterio de la Iglesia 


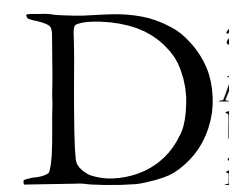
ans un des derniers chapitres du second livre du Tratado llamado leche de la fe del príncipe christiano, Luis de Maluenda s'adresse directement à son lecteur dans un bref dialogue à propos de la mort des martyrs. Devant un interlocuteur auquel il prête l'opinion inverse, il affirme « Yo digo que no puede morir mala muerte el que bien vive ». La raison en est bien une confusion entre visible et invisible, une erreur d'optique, au sens concret du terme, en quelque sorte. Il ajoute: «Si miras las muertes de los mártires con los ojos de la carne dirás que murieron malas y crueles muertes, mas pregunta a los ojos de la fe y dezirte han preciosas que son las muertes de los sanctos en los ojos de Dios. ${ }^{1} \gg$ C'est un lieu commun théologique ${ }^{2}$ : les yeux de la chair ne sont pas adaptés pour contempler l'œuvre d'un Dieu que le Symbole de Nicée-Constantinople définit, dans son $5^{\mathrm{e}}$ article, comme "creator visibilium omnium et invisibilium». Le chrétien doit donc se détacher d'un réel visible qui fait obstacle à son salut au moyen de la seule considération, en son âme, d'un invisible tout aussi réel. Si le but de cette quête spirituelle, la vision béatifique, est unique, les chemins pour y parvenir sont, quant à eux, multiples.

Le franciscain de Burgos qui est l'auteur de ce traité nous est très mal connu. S’il n'avait signé cet ultime opus, son propre nom ne serait peut-être jamais parvenu jusqu'à nous, puisqu'il était de tradition, chez les auteurs appartenant à l'Ordre des Frères Mineurs, de demeurer anonymes dans un souci d'humilité. Sans la sagacité d'Eugenio Asensio, ce traité, dont il ne subsiste que deux exemplaires, l'un à la Bayerishe Staatsbibliothek de Munich et l'autre à la Bodleian Library ${ }^{3}$, pourrait très bien avoir continué à échapper aux chercheurs ${ }^{4}$. Les éléments autobiographiques disséminés dans les premières pages de ce texte nous enseignent qu'avec sa rédaction Maluenda répond aux sollicitations d'un mystérieux religieux, dont il ne dévoile pas l'identité, tout en accomplissant un vœu fait à la Vierge. Tombé malade le 8 septembre 1544, jour de la solennité de la nativité de NotreDame, Maluenda, déjà très âgé, avait recouvré la santé à la fin de l'octave de cette même fête ${ }^{5}$. Pour cette raison sans aucun doute, ce traité semble écrit à la hâte et d'une seule

\footnotetext{
${ }^{1}$ Maluenda, Luis de, Tratado llamado leche de la fe del príncipe christiano. Con LXII milagros de Jesu Christo nuestro Dios y redemptor, y con los mysterios del Antechristo, y con las ropas de las virtudes morales y theologales, copilado de los excelentes libros por fray Luys de Maluenda, a los quarenta años de ávito de religioso menor, y examinado por el reverendísimo Padre ministro general, y aprovado por el Consejo Real, Burgos, Juan de Junta, 1545.

${ }^{2}$ Maluenda médite ici sur le psaume 123 qui représente serviteurs et servantes, le regard dirigé vers leur maître et leur maittresse. Peut-être s'inspire-t-il d'un commentaire de ce même psaume, comme celui d'Hilaire de Poitiers qui distingue yeux de la chair et yeux de la foi. Cette référence contient un point de controverse dès lors qu'est posée la question de la connaissance ultime de Dieu. La vision béatifique se fera-t-elle au moyen des yeux de la chair ou de ceux de l'entendement?

${ }^{3}$ Nous avons utilisé l'exemplaire de Munich. Le Tratado llamado leche de la fe del príncipe christiano figure dans l'Index espagnol de 1559 et, à en juger par le nombre d'exemplaires qui ont survécu, il semblerait que la répression inquisitoriale contre cet ouvrage ait été particulièrement efficace, cf. BUJANDA, José María de, Index de l'Inquisition espagnole, 1551, 1554, 1559, Genève, Droz, 1984, p. 199 et 504. Quoi qu'il en soit, ce très volumineux in-folio ne paraît pas avoir connu un tirage aussi important que les deux in-quarto attribués à Maluenda. L'inventaire de l'imprimerie de Juan de Junta, réalisé en 1556, fait état de 40 exemplaires du Tratado llamado leche de la fe del principe christiano, tandis que le Tratado llamado de las excelencias de la fe et le Vergel de virginidad ont été respectivement tirés à 289 et 201 exemplaires, cf. PETTAS, William, A Sixteenth-Century Spanish Bookstore: The Inventory of Juan de Junta, Philadelphia, American Philosophical Society, 1995, p. 119, 126 et 147. 4 AsEnsio, Eugenio, «Fray Luis de Maluenda, apologista de la Inquisición, condenado en el índice inquisitorial », in Arquivos do Centro Cultural Português, IX (1975), p. 87-100.

5 «la ocasión fundamental por la qual este tratado se copiló fue ésta, que como este pecador estoviese muy doliente el día del nascimiento de la Virgen y madre de Dios prometió a la Virgen diziendo, que si fuese servida de darme salud que copilaría un tratado en reverencia de su precioso Hijo, y el día viii de la virgen por la piedad de la Virgen quitóseme la enfermedad y también seguramente, se copilaron estos papeles por importunación de un religioso teólogo, que muchas vezes gastó zelosas importunaciones, para que antes que
} 
traite, l'auteur indiquant parfois que certains passages doivent être replacés bien avant l'endroit où on les rencontre dans le texte. Dédié au futur Philippe II, cet ouvrage polymorphe est un centon d'Écritures saintes et d'exégèses diverses. Il combine, après un prologue long de quelque trente-quatre pages in-folio densément remplies, et sur plus de cinq cents folios imprimés sur deux colonnes, miroir du prince, Vita Christi, manuel de spiritualité ascétique et traité eschatologique sur la venue de l'Antéchrist. Son titre intégral est le suivant :

Leche de la fe del príncipe christiano. Con 62 milagros de Jesu Christo nuestro Dios y Redemptor. Y con los mysterios del Antechristo : y con las ropas de las virtudes morales y theologales. Copilado de los excelentes libros por fray Luys de Maluenda. A los quarenta años de ávito de religioso menor. Y examinado por el Reverendíssimo Padre ministro general : y aprovado por el Consejo Real.

Ces quelques lignes rendent pleinement compte de l'une des caractéristiques les plus frappantes du style de Maluenda, tout particulièrement dans le très long prologue du traité Leche de la fe del principe christiano. Véritable traité au seuil du traité, il sera l'objet principal de notre étude, bien que ce trait significatif apparaisse dans l'ensemble de l'ouvrage comme dans le reste de la production de ce franciscain. À la lecture de ces quelques lignes du titre, il est indéniable que le castillan dans lequel il s'exprime, aux antipodes du raffinement de la prose humaniste de certains de ses contemporains, manque singulièrement d'élégance. Il n'en reste pas moins extrêmement savoureux par l'abondance des images auxquelles Maluenda a recours et en raison de la banalité - trivialité pourra être tenté de dire parfois le lecteur de notre temps - de ces mêmes images : ces "ropas de las virtudes morales y theologales ${ }^{6}$ » en sont un premier exemple. Il n'y a rien de surprenant, a priori, à voir un des principaux procédés mis à l'honneur dans les textes sacrés et dans ceux des saints et docteurs apparaittre dans les écrits des auteurs spirituels. Théologiens, spirituels, orateurs sacrés, ont pieusement recueilli ce legs stylistique et rhétorique tant dans les commentaires les plus rigoureux des Écritures que dans les méditations les plus libres. Maluenda l'assume sans ambages, dans le premier chapitre de son traité :

... el Espíritu sancto que guió en las sanctas Escripturas la pluma de los profetas puso diversas comparaciones y semejanzas de cosas corporales, como escaleras para subir a los altos sentidos de las virtudes espirituales, y los sanctos doctores de la Iglesia usaron en sus sanctos libros de semejanzas y comparaciones para dar a sentir las verdades divinas e invisibles. Y el Redemptor, maestro de la soberana verdad, usó en su altísima doctrina de semejanzas y comparaciones y parábolas de cosas visibles y llanas como son viña y vid, y simiente y trigo y grano de mostaza, y de candela, y de candelero, y de sal, y de ciudad, y de redes para pescar, y de peces, y de tesoro, y de dineros, y de perlas, y de otras muchas y muy llanas comparaciones y semejanzas, como de escaleras para subir a las verdades divinas y espirituales que en su altísima dotrina enseñava. ${ }^{7}$

se acabase la candela de la vida deste pecador, que para provecho común escribiese algunos misterios de los sagrados evangelios y de la vida de Jesucristo ... ", MALUENDA, Tratado llamado leche de la fe del príncipe christiano, op. cit., fol. C ii r-v.

${ }^{6}$ C'est nous qui soulignons.

${ }^{7}$ Ibid., fol. ii v. 
En la matière, l'œuvre de Luis de Maluenda est remarquable car le discours imagé l'est jusqu'à l'excès. En effet, les images verbales ou discursives ${ }^{8}$ convoquées couvrent, d'une part, un champ de l'expérience humaine éminemment vaste et varié. D'autre part, leur enchaînement est, pour ainsi dire, inexorable, à tel point qu'on peut s'interroger, in fine, sur leur véritable statut au sein du discours spirituel de Maluenda. Certes, comme le souligne le recours à l'inépuisable métaphore scalaire, c'est par les choses visibles, per visibilia, qu'il est possible d'accéder à ce qui est invisible, ad invisibilium, selon un processus conjoint d'ascèse et d'élévation. La piètre qualité littéraire de cette ultime production pourrait ainsi conduire à penser qu'il ne s'agit pas d'autre chose que d'une manifestation, répandue et légitime, mais hypertrophiée, d'un procédé d'écriture spirituelle vieux de plusieurs siècles à l'époque où Maluenda écrit. Toutefois, la lecture de quelques-unes de ces pages suggère fortement que l'abondance des images a partie liée, non seulement avec toute une école de spiritualité fort traditionnelle, mais aussi avec les orientations spirituelles de leur auteur. Bien que consacré par toute une tradition exégétique à laquelle Maluenda est amplement redevable, le visible qui envahit chaque recoin de son propos spirituel configure un monde plein de vie, et non pas fossilisé sous l'effet d'une caution patristique ou théologique pourtant revendiquée. Il constitue donc un puissant trait d'union entre l'auteur, sa doctrine spirituelle, et le lecteur. Aussi, par-delà l'étonnement que provoque chez le lecteur contemporain la hardiesse de certaines images, dont nous ignorons le véritable impact sur l'homme du premier XVI ${ }^{\mathrm{e}}$ siècle, il faut bien reconnaître que notre champ d'investigation n'est pas uniquement métaphysique et théologique mais également sociologique. C'est bien le rapport d'un homme - Maluenda - et des hommes aux images qui est en jeu. Le maillage très serré que les images tissent à travers toute l'œuvre n'a sans doute d'autre fin que de s'ériger en instrument du contrôle de la méditation spirituelle du dévot.

\section{De l'obscurité des tombeaux aux images en cascade : ascétisme et catéchèse dans le Tratado llamado leche de la fe del príncipe christiano}

Les images se bousculent sous la plume de Maluenda, non seulement dans le dernier de ses traités, mais dans son œuvre tout entier. Au sein de cette production, l'une d'elle revêt une importance particulière puisqu'on la retrouve dans le paratexte du premier de ses traités et dans l'ultime ouvrage publié peu avant sa mort sans doute. Elle se présente déclinée de deux manières différentes dans le Vergel de virginidad, traité dont le colophon

\footnotetext{
8 Nous regrouperons dans cette unique catégorie les «semejanzas y comparaciones y parábolas de cosas visibles » auxquelles Maluenda fait allusion. Nous ne proposerons pas de typologie des procédés rhétoriques mis en œuvres, si bien que nous employions, de manière indiscriminée, les étiquettes «image » et «métaphore» pour désigner tous les dispositifs discursifs visant à produire, dans l'esprit du lecteur, la formation d'une image mentale. Les notions d'image et de ressemblance possédaient à l'époque médiévale des limites diverses selon les auteurs. Nous nous en tiendrons donc à la prudente définition proposée par Robert JAVELET, Image et ressemblance au XII siècle, De saint Anselme à Alain de Lille, Paris, Letouzey-Ané, 1967, t. I, p. XIX-XXIII, qui ne retient que la relation de similitude. Elle présente l'avantage, comme le rappelle MarieLaure SAVOYE, De fleurs, d'or, de lait, de miel: les images mariales dans les collections miraculaires romanes du XIII ${ }^{e}$ siècle, thèse sous la direction de François Lecercle, soutenue à l'Université Paris-Sorbonne en juin 2009, p. 14, de situer l'image «dans un entre-deux, dans un va-et-vient du corporel au spirituel que la mise en récit des perceptions visuelles ne fera que rendre plus évident. »
} 
nous indique que s'il fut publié en 1539, sa rédaction était déjà achevée en $1532^{9}$, et dans Leche de la fe del principe christiano, quelque treize ans plus tard. Ces deux variations sur un même thème illustrent, de manière quelque peu surprenante, la dialectique entre le visible et l'invisible et en souligne le caractère fondamental dans la démarche spirituelle de Maluenda. Leur développement est original bien que leur fondement repose sur un passage de saint Jérôme tel que le signale l'auteur - sans que la référence précise ne soit indiquée toutefois ${ }^{10}$. Le Père de l'Église exalte tout le bénéfice que les vivants retirent des œuvres de ceux qui sont ensevelis sous terre, cachés aux yeux des hommes donc. Dans Leche de la fe del principe christiano, ce sont les actes hérö̈ques de la Reconquête, trabajos, au sens étymologique du terme, de non moins hérö̈ques princes castillans, qui fondent le repos dont Maluenda et ses contemporains jouissent :

Razón muy grande tuvo el divino San Hierónimo quando dixo que los trabajos de los muertos y de los enterrados son descansos y regalos de los bivos, que gozan de sus trabajos y sudores, como está muy clara esta verdad en los trabajos que gastaron los príncipes de Castilla en conquistar los reynos de Toledo y de Córdoba y de Sevilla y de Granada, y en ganar los otros reynos de los poderíos de los bárbaros y de los moros, los quales reynos y señoríos ganados con los afanes y trabajos sangrientos de los príncipes pasados y muertos, son agora descansos y regalos de los bivos, que gozan de los reynos y señoríos que los trabajos de los muertos conquistaron y ganaron con sus trabajos bañados en sudores de sangre ${ }^{11}$.

Prenant en exemple le comte Fernán González, dont l’histoire, enterrée pendant près de cinq cents ans selon Maluenda, vient d'être publiée ${ }^{12}$ au moment où il rédige le prologue du Vergel de virginidad, ce dernier texte se montre plus explicite puisqu'il invite également ses lecteurs à déterrer les véritables vivants, ces bienfaiteurs de la chrétienté que sont les saints et docteurs, leurs livres et, plus encore, les Évangiles.

Y como quando los cuerpos sanctos se desentierran salen suavísimos olores de suavidad así quando los tratados y los libros de los santos aunque sean copilados por manos de pecadores, como son las deste su siervo pecador, se leen y se estudian salen unos olores celestiales para consolación de la vida cristiana como destos tratados leídos y usados redundarán olores angélicos, castos y virginales a quien los leyere y tratare. ${ }^{13}$

\footnotetext{
${ }^{9}$ « Acabáronse los presentes tratados. Compuestos en san Francisco de Salamanca. Año de mil y quinientos y treynta y dos.

A gloria y alabança de Jesucristo nuestro Dios, y de su gloriosa madre hazen fin los presentes tratados llamados Vergel de virginidad y Mysterio de los ángeles. Compuestos por un religioso de la orden de los menores. Fueron impresos en la muy noble y más leal ciudad de Burgos, en casa de Juan de Junta. Acabóse a dos días del mes de junio, año de mil y quinientos y treynta y nueve años. ", MALUENDA, Luis de, Vergel de virginidad con el edificio espiritual de la caridad, y los mysterios de la Virgen sin par. Y otro tratado de los mysterios de los ángeles. Con treze servicios que haze el Ángel custodio. Compuesto por un religioso de los menores de la provincia de Santiago, Burgos, Juan de Junta, 1539, sans foliation [fol. $r$ ii r]

${ }^{10}$ Nous n'avons pas retrouvé l'origine de cette référence.

${ }^{11}$ Maluenda, Luis de, Tratado llamado leche de la fe del principe christiano, op. cit., sans foliation [A i r ].

12 Miguel Avilés Fernández, Los «varones espirituales », una corriente reformadora en la España de los erasmistas y los alumbrados, thèse de doctorat en histoire moderne soutenue en 1974 à l'Universidad Autónoma de Madrid, p. 134, a identifié ce texte comme pouvant être la Chrónica del noble Cavallero el conde Fernán González, Burgos, Juan de Junta, 1530, ou bien la Historia breve del excelente cavallero el conde Fernán González, sacada del libro viejo que está en el Monasterio de Sant Pedro de Arlanza, Burgos, Juan de Junta, 1537.

13 MALuEnda, Luis de, Vergel de virginidad, op. cit., fol. ii r.
} 
Cette seconde déclinaison, dans sa dimension synesthésique, souligne l'autoréférentialité et le caractère métadiscursif du propos de Maluenda. Ce dernier assure lui-même la promotion de son ouvrage sans déroger à une humilité fondamentale garantie par le rôle de compilateur qu'il s'est assigné. Mais là n'est pas l'essentiel. Bien sûr, ce qui est enseveli, caché aux yeux des hommes de son temps, doit être révélé, tout comme le Nouveau Testament dévoile ce qui est caché dans l'Ancien. Il ne faudrait toutefois pas se méprendre sur ce que ces quelques mots du début du Vergel de virginidad mettent véritablement au jour. C'est bien une lamentation sur les maux spirituels du temps qui est sous-entendue ici. Elle n'a rien de bien original, encore moins chez un franciscain qui participe pleinement des courants messianiques et eschatologiques qui sont à l'œuvre, depuis déjà longtemps, chez nombre de ses frères en religion ${ }^{14}$. En effet, le lecteur est invité à comprendre que, contre des fossoyeurs des trésors de la spiritualité chrétienne que l'auteur ne nomme pas encore dans ces deux prologues, ce même auteur entend imiter, avec une sainte morbidité, l'exemple de saint Jérôme, grand lecteur de «muchos libros excelentes de los filósofos y horadores muertos y enterrados» et de "muchos libros de dotores christianos griegos y latinos muertos y enterrados bivos ${ }^{15}$ »

Le prologue de Leche de la fe poursuit son exploration de la dialectique entre visible et invisible, pour peindre, sous les espèces du ver à soie, ces hommes qui s'ensevelissent euxmêmes pour mieux exhumer une sainte doctrine. Didyme, ce disciple d'Origène traduit par saint Jérôme, en est un paradigme. Aveugle de naissance, prisonnier d'une obscurité semblable à celle du tombeau, incapable d'embrasser du regard la création, il eut le privilège de recevoir la science infuse et d'enseigner les Écritures sans jamais les avoir lues, "como gusano de seda muy diestro y muy sabio en labrar sirgos y sedas. ${ }^{16} \gg$ Les docteurs de l'Église, Jérôme, Ambroise, Augustin, Grégoire, Jean Chrysostome, Origène, saint François et saint Dominique, pour ne tirer que ces noms d'une assez longue liste, sont également à l'image des vers à soie. Ils naissent et grandissent contre les seins virginaux ${ }^{17}$ et se nourrissent des feuilles du mûrier. L'analogie entre le cocon de soie dont s'entoure ces chenilles et le retrait du monde des Pères et docteurs de l'Église pour étudier les textes sacrés fonctionne à merveille ici. L'image devait être familière à un lectorat relativement large et proposait une illustration assez simple d'un propos ascétique rebattu ${ }^{18}$.

\footnotetext{
14 Sur cette question, cf. Milhou, Alain, Colomb et le messianisme hispanique, Montpellier, ETILAL, 2007, notamment p. 319-320. Ce messianisme est pleinement assumé dans le Tratado llamado leche de la fe del príncipe christiano. Son titre délivre l'organisation interne de la plupart de ses soixante-cinq chapitres. Ils possèdent tous une organisation tripartite : Maluenda commence par y exposer la thématique qui est propre à chacun d'eux, continue en faisant le récit d'un miracle du Christ et termine par la présentation d'une ou plusieurs préfigurations de l'Antéchrist.

${ }^{15}$ Maluenda, Luis de, Tratado llamado leche de la fe del principe christiano, op. cit., sans foliation [a i v].

16 Ibid., A ii v.

${ }^{17}$ Il s'agit d'une référence à la technique archaïque de l'utilisation de la chaleur humaine pour l'incubation des graines.

18 La culture du ver à soie et du mûrier avait été introduite dans certaines zones de la vallée de la Sierra Nevada, dans le royaume de Grenade, dès les années 740, cf. LAGARDÈRE, Vincent, « Mûrier et culture de la soie en Andalus au Moyen Âge (Xe-XIVe siècles) », Mélanges de la Casa Velárquez, 26 (1990), 97-111. Au XVI ${ }^{\mathrm{e}}$ siècle, la sériciculture était relativement répandue en Castille et en Aragon. Tolède, Séville, Murcie et Valence, étaient des centres importants de cette production textile. Bien que sa culture ne couvrît pas la totalité du territoire, son existence et ses caractéristiques pouvaient être connues par les hommes et les femmes du XVI siècle. Thérèse d'Avila nous en apporte la preuve. Au chapitre II de la cinquième partie des Moradas, l'image du ver à soie est utilisée par la sainte qui confesse n'en avoir jamais vu en réalité. On peut lire en effet : «con el calor, en comenzando a haver hoja en los morales, comienza esta simiente a vivir ; que hasta que hay este mantenimiento de que se sustentan está muerta ; y con hojas de moral se crían, hasta que después de grandes, les ponen unas ramillas, y allí con las boquillas van de sí mesmos hilando la seda y hacen unos capuchillos muy apretados, adonde se encierran; y acaba este gusano, que es grande y feo, y sale del mesmo capucho una
} 
De fait, les nombreuses images triviales qui parcourent le Tratado llamado la leche de la fe del príncipe christiano, semblent former un véritable liber idiotarum, un livre d'images verbales à proprement parler, au même titre que les représentations iconographiques qui ornaient, sous diverses formes, les églises. Les plus simples des lecteurs de Maluenda trouvaient donc dans le traité tout un arsenal d'iconographie mental dont l'intelligence était parfaitement à leur portée et qui rendaient visible et presque palpable un propos relativement abstrait parfois. Le traitement de l'image de la gargouille est un autre exemple de cette dimension figurative du texte ${ }^{19}$. Les gargouilles représentent tous les auteurs spirituels qui canalisent et orientent la pluie de la doctrine des saintes Écritures et des textes pieux vers les chrétiens. L'abondance et la pureté des eaux qu'elles déversent sont proportionnelles à la moralité de la vie des auteurs de ces écrits. Ainsi, les philosophes, les païens et les hérétiques sont des gargouilles qui déversent une eau impropre à étancher la soif des cœurs des chrétiens. Enfin, Maluenda fait de cette métaphore un miroir de son propre travail pour offrir à son lecteur un curieux autoportrait.

Y destas canales de tejados ay muchas en la Yglesia, y una dellas y bien seca y bien vazía, es este pecador que estas letras aquí nota, que con grande afrenta y berguença confiesa este pecador aquí esta culpa que sirve en la Yglesia de canal y tejado seca y vazía, y sirven otros muchos a Jesucristo y a la Yglesia de pilas llenas y de canales llenas de vidas virtuosas y de dotrinas para los ganados de Jesucristo ${ }^{20}$.

La profusion du recours aux images et leur inexorable enchaînement dans plusieurs passages importants du traité trahissent une certaine boulimie chez l'auteur. Maluenda les traque dans les trésors de la littérature religieuse, les adapte à son propos spirituel le cas échéant. Une certaine prédilection pour les métaphores concrètes, tirées de la vie quotidienne est perceptible, en outre, dans le recours à certaines images originales. La concaténation métaphorique qui soutient l'économie du long prologue de Leche de la fe est symptomatique, par ailleurs, d'un rapport compulsif à leur insertion dans le discours spirituel, le passage d'une image à l'autre se faisant par libre association d'idées. Ainsi, le cheminement peut parfois sembler quelque peu erratique. Comme on l'a vu, Maluenda commence par évoquer le ver à soie, pour exploiter ensuite des images tirées, de près ou de loin, du mûrier. Rompant momentanément le fil de la métaphore, le silex prend le relais du ver à soie puisqu'il représente l'inflexibilité face aux tentations de ce monde et la détermination à s'en maintenir éloigné. Il est tout autant un symbole de la charité. Le cocon $\mathrm{du}$ ver est ensuite une figuration du tombeau, tout comme le puits est implicitement présenté comme la sépulture des flots des savoirs profane et sacré. L'eau qu'on y puise peut s'avérer saumâtre. Elle est pure quand elle tombe du ciel, et les auteurs spirituels capables de la recueillir et de la transmettre aux hommes sont les gargouilles humides et donc fécondes auxquelles nous venons de faire référence. Il ne s'agit là que d'un aperçu d'un procédé récurrent tout au long du Tratado llamado leche de la fe del príncipe christiano. Le recours

mariposica blanca muy granciosa. », SANTA TERESA DE JESUS, Moradas del Castillo interior, in Obras completas, Madrid, Biblioteca de Autores Cristianos, 1954, t. 2, p. 398. Le développement de cette image illustre la totalité du cheminement ascétique qui conduit jusqu'à l'expérience mystique puisque l'éclosion du papillon représente l'envol d'une âme gracile une fois libérée des entraves terrestres d'un corps nécessairement grossier, à l'image du ver en son cocon.

${ }^{19}$ Il est vrai toutefois que l'image apparaît en Proverbes, 27, 15: «Gargouille qui ne cesse de couler un jour de pluie et femme querelleuse sont pareilles !»

20 Maluenda, Luis de, Tratado llamado leche de la fe del principe christiano, op. cit., sans foliation [A vii v, par déduction]. 
à la métaphore est donc un procédé central dans le langage utilisé par Maluenda. L'énumération des premières images du traité indique également qu'elles sont au service d'un discours ascétique dans la plus pure tradition médiévale. Il est lui-même figuré dans l'impérieuse catabase de la descente dans les profondeurs de la terre, dans le monde invisible, pour rechercher les vivants qui y gisent et les exhumer. Ainsi, des saints docteurs qui

después que se sepultaron sus propias pasiones enterrando la sobervia, y la invidia, y la vanagloria, y la avaricia, y la gula y sobre todo el vicio carnal que es enemigo mortal, que embaraça los sentidos espirituales de las santas Escripturas, entraron en la sepultura rica y honrada de la continua consideración y devoción de la vida de Jesucristo, y dando su razón y entendimiento y memoria a la devoción reverencial de Jesucristo, y dándose todas enteras a la vida virtuosa y espiritual, y enterrándose como gusanos de seda en sus sepulcros de seda... ${ }^{21}$

$\mathrm{Au}$ terme de cette purgation des vices vient ainsi la promotion des vertus, qui arrivent en cinquième position dans la liste de ce que doit cultiver l'homme pour imiter l'exemple du ver à soie. Cette "escalera espiritual de las virtudes de Jesucristo », très librement inspirée de l'Échelle sainte de saint Jean Climaque, est particulièrement mise à l'honneur peu avant le début du premier chapitre du premier livre. Elle s'y présente sous la forme d'une table récapitulative, qui suit le sommaire du livre, et qui tient lieu d'aidemémoire puisqu'elle ne renvoie à aucune division de l'ouvrage et ne contient pas davantage d'indices de localisation.

\section{Docere ou delectare? Une fausse alternative}

La prolifération des images dans le discours spirituel du Tratado llamado leche de la fe del principe christiano est loin, toutefois, de répondre à une seule finalité catéchétique. Voilà, du moins, ce que donnent à penser les présentations successives de l'ouvrage au cours du prologue. Maluenda revient en effet à trois reprises sur la nature de son traité. La métaphore du lait qui donne son titre à l'ouvrage représente explicitement «la fe y las dotrinas de la fe ", contenues dans les livres saints et pieux. D'après cette métaphore empruntée à saint Augustin ${ }^{22}$, dans Leche de la fe del príncipe christiano

se contienen los misterios principales de la vida reverencial de Jesucristo y sus dotrinas y sus palabras santísimas y los milagros principales que relatan los sagrados evangelios, los quales son las fuentes y mineros de la fe christiana como los pechos de la madre son fuentes de la leche del infante. (...) Y estos papeles aunque llana y baxamente rimados son fuentes de leche de la fe christiana para todo linaje de personas, por aver en ellos muchas dotrinas de los santos dotores jamás vistas en estilo castellano ${ }^{23}$.

Les préventions que Maluenda nourrit à l'égard de ses talents littéraires tiennent, à un premier niveau d'analyse, au fait qu'il ne s'exprime pas en latin puisqu'il traduit et compile

${ }^{21}$ Ibid., fol A iv v.

22 Homélies sur l'Évangile de Jean, tract. XLVIII, 1.

${ }^{23}$ Ibid., sans foliation [B vi v]. C'est nous qui soulignons. 
extraits des évangiles et passages d'exégèses diverses. La suprématie du docere, de la transmission d'une doctrine traditionnelle au surplus, n'a rien qui puisse susciter l'étonnement dans ce type d'ouvrage. Une exubérante revendication du delectare peut paraître moins habituelle en revanche :

Y de aquí es que el presente tratado servirá al corazón christiano que en él se ocupare, de nido de ave fénix, compuesto de las ramas olorosas a la primera vista algo bastas de los dezinueve (sic) capítulos primeros, en los quales se tratan (sic) de las diferentes leches y dotrinas y muchas historias de paganos muy sabrosas y olorosa, como la historia de los dos dragones y la historia de la donzella criada con leche de dragones y la historia, digna de ser escripta con letras de oro, de la calumnia. Y la historia de Judas el traydor con los misterios que los santos dotores escrivieron de Judas, y con otras muchas historias y misterios de los santos jamás vistos en estilo castellano...24

À l'importante composante exégétique du traité viennent s'ajouter ces « historias de paganos muy sabrosas y olorosas » qui font prendre au discours de Maluenda des accents publicitaires, déjà perceptibles, dans un autre registre, dans la revendication du caractère inédit en langue vernaculaire de certains commentaires bibliques. Tout se passe comme si, au seuil de cette somme, l'auteur éprouvait la nécessité de rassurer son lecteur en précisant que la gravité et l'austérité de sa matière principale seront tempérées par des passages plus légers. Le choix des épithètes "sabrosas y olorosas » n'est pas anodin : la métaphore de l'assaisonnement de la nourriture spirituelle était déjà courante au Moyen Âge. On la retrouve dans les années 1560 dans l'introduction que François Borgia rédige pour ses méditations sur les évangiles de l'année liturgique. Les images y sont explicitement présentées comme le principal condiment pour ces mets au goût amer parfois pour certains $^{25}$. Le futur préposé général de la Compagnie de Jésus a ici à l'esprit de véritables images formant un apparat iconographique qui vient compléter le texte ${ }^{26}$. Il y a tout lieu de penser que les nombreux procédés métaphoriques présents de Leche de la fe del príncipe christiano sont à compter au nombre des ingrédients qui rendent l'ingestion de la nourriture spirituelle plus facile ${ }^{27}$. Dans ce dernier ouvrage, Maluenda se montre particulièrement ingénieux pour mettre en image son propos spirituel. Nous en voulons pour preuve cette nouvelle exploitation de la métaphore de l'eau saumâtre qui révèle désormais son utilité.

\footnotetext{
${ }^{24}$ Ibid., fol. C r.

25 « Pour plus de facilité dans la méditation, on se propose une image qui représente le mystère de l’Évangile ; et l'on regardera l'image avant de commencer la méditation ; et l'on sera particulièrement attentif à ce que l'on devra observer à nouveau dans le courant de la méditation, pour en tirer le plus grand profit. Parce que la fonction de l'image est comparable à la préparation d'un aliment que l'on doit manger, de manière qu'on ne puisse que le manger. S'il en allait autrement, l'esprit devrait s'efforcer par les seuls mots de se représenter l'objet de la méditation, à sa charge et à grand peine. ", cité dans DEKONINCK, Ralph, Ad imaginem. Statuts, fonctions et usages de l'image dans la littérature spirituelle jésuite du XVII siècle, Genève, Droz, 2005, p. 156. Cf. également LUBAC, Henri de, Exégèse médiévale, Paris, Cerf, 1993, tome I, 1, p. 119.

${ }^{26}$ Le Tratado llamado leche de la fe del principe christiano n'en contient qu'une à proprement parler, en regard de la page de titre : une xylographie représentant, cela va de soi, une Vierge allaitant l'enfant Jésus, assise sur un trône devant lequel est agenouillé un jeune prince en position d'orant, épée à la ceinture et couronne et sceptre déposés aux pieds de la Vierge à l'enfant. Ainsi, le rôle de cette gravure semble se cantonner à celui d'illustration du titre afin de souligner que ce traité de spiritualité est tout à la fois un miroir du prince. Les lettrines placées en tête de chapitre, ne présentent pas d'intérêt particulier à première vue.

${ }^{27}$ En la matière, la pensée de Maluenda a évolué. En 1539, dans le Tratado llamado excelencias de la fe, il associait « la salsa verde », cuisinée à base de persil, à Judas et à l'hérésie en général : "¿Quién podrá sumar y contar las salsas y peregiles de las buenas obras exteriores, que los ereges han guysado en la Yglesia, para que se puedan comer sus condenadas doctrinas con gusto y sabor? ", Maluenda, Luis de, Tratado llamado excelencias de la fe, op. cit., sans foliation [fol. c ii r].
} 
Indigne des beaux flacons dans lesquels on verse l'eau pure ou l'eau de rose, elle permet malgré tout de mettre à l'épreuve la qualité de la teinture d'un vêtement, comme le rappelle Maluenda au chapitre XXXII. En somme, la Providence n'a rien voulu d'inutile dans l'ensemble de la création. Cette eau salée est également celle qu'on utilise

para regar y refrescar las casas cristianas de las historias de los paganos así griegas como latinas jamás vistas en romance sacadas con los braços muertos y cansados de los libros de los paganos como de pozos de aguas salobres para refrescar los ojos de los letores ${ }^{28}$.

Ainsi, dans une curieuse mise en abyme, la désignation même du procédé consistant à insérer des apologues et anecdotes profanes qui rendent la lecture plus amène est un élément métadiscursif qui fonctionne de manière identique, par métaphorisation, puisqu'il repose sur la figuration d'une abstraction. Les images, verbales ou discursives, ont partie liée, en outre, avec une mise en texte d'un propos général « baxa y llanamente rimado[s] ${ }^{29}$ », dépourvu de recherche et d'ingéniosité stylistique ${ }^{30}$. Marquées par la familiarité du quotidien face au sublime des vérités de la foi et de leur expression directe sous l'inspiration de l'Esprit saint, elles ne leur apportent pas moins une saveur particulière tout aussi essentielle aux yeux de Maluenda. Par le discours imagé, l'auteur donne un piquant particulier à certains passages. Il soutient ainsi l'attention de son lecteur en s'exprimant souvent cum grano salis.

Cependant, ces considérations rhétoriques et stylistiques ne prennent toute leur envergure que si l'on veut bien prêter attention au caractère polémique dont elles sont porteuses de manière plus ou moins explicite. L'humilité de Maluenda est également intellectuelle. Plus qu'un regret, la reconnaissance du caractère prosaïque du discours de Leche de la fe del principe christiano est une revendication de la simplicité contre l'élégance et le raffinement outranciers des humanistes tout d'abord. La mécanique des fluides (lait, eaux et vin) qui sous-tend une bonne part du texte dévoile cette intention critique, pour ne pas dire apologétique. Glosant un passage du cinquième chapitre du Cantique des cantiques, Maluenda oppose le sein virginal d'où coule le lait de la sainte doctrine aux «tabernas de vino de las escuelas de la retórica y eloquencia de los retóricos y de los oradores » et, un peu plus loin, aux « tabernas de la eloquencia mundana de Tulio (...) de Platón (...) de las escuelas de los grandes filósofos y oradores ${ }^{31} »$ Dans une même métaphore, l'élégance stylistique des humanistes et le savoir antique qu'ils contribuent à ressusciter se trouvent récusés. Alors qu'il serait tentant de faire de cet adversaire des humanistes un tenant de la scolastique, Maluenda adopte une posture qui lui fait rejeter tout autant l'apport de la logique nominaliste en théologie, monstruosité produite par une scolastique médiévale arrivée au dernier stade de la dégénérescence. En somme, étude des langues, des sciences, des arts libéraux (sans discrimination entre les disciplines du trivium, comme la rhétorique, et du quadrivium, comme la logique), de la nécromancie ou de l'astrologie, n'ont droit de cité dans les ouvrages de spiritualité de ce franciscain ${ }^{32}$. On reconnaît même à leur maîtrise les

\footnotetext{
${ }^{28}$ Maluenda, Luis de, Tratado llamado leche de la fe del principe christiano, op. cit., sans foliation [A viii r].

${ }^{29}$ Cf. supra.

30 Telle est la signification première de son étymon latin, rimor, ari.

${ }^{31}$ Maluenda, Luis de, Tratado llamado leche de la fe del príncipe christiano, op. cit., fol. A iii v.

32 Comme en beaucoup d'autres endroits de ce traité, Maluenda livre le contenu d'une prophétie selon laquelle, au moment de la venue de l'Antéchrist, «avrá por todo el mundo estudios de todas las lenguas y de todas las sciencias y artes liberales, y mucho más avrá grande abastança de letras de nigromancia y de astrología, y de lógica, y de retórica », ibid., fol. cclviii r.
} 
ministres de l'Antéchrist: ils seront plus savants que Platon et Aristote et plus grands orateurs que Cicéron et Démosthène selon certaines prophéties ${ }^{33}$.

Dans la pratique, la position de Maluenda est plus nuancée, ou du moins se montre-til plus souple que ce qu'il veut bien laisser croire à son lecteur. Les passages ne manquent d'ailleurs pas où il puise dans le trésor des sentences tirées de Platon et d'autres philosophes ou auteurs antiques. Point de contradiction toutefois, car ces occurrences de ce qu'il a semblé vouloir bannir n'engagent en rien les choix théologiques qui sont ceux du Tratado llamado leche de la fe del principe christiano, mais également du Vergel de virginidad, du Misterio de los Ángeles ou encore du Tratado llamado de las excelencias de la fe. Dans ces textes, le discours théologique et spirituel ne repose pas sur des définitions exprimées sur le mode logique. Il est principalement fondé sur l'exploitation d'analogies, de similitudes, plus ou moins arbitraires, entre vérités de la foi chrétienne et éléments de la vie quotidienne. Il ne se situe pas de plain-pied dans le champ de la théologie symbolique, qui exploite des images autrement complexes tels les hiéroglyphes, mais prépare peut-être la voie vers ce domaine ${ }^{34}$. L'auteur spirituel soucieux de l'orthodoxie de son propos - laquelle ne tardera pas à être mise en doute par l'Inquisition - devait trouver dans cette théologie de l'image si basique un précieux garde-fou contre les dérives qu'il pouvait constater de ses yeux en ce premier $\mathrm{XVI}^{\mathrm{e}}$ siècle. Le bénéfice que le lecteur retirait de ce parti pris ne devait pas être négligeable non plus.

\section{Métaphores mortes, spiritualité vive?}

La revendication de la coexistence du docere et du delectare dans le recours à l'image, au sein d'un texte portant sur de graves sujets, est en effet révélatrice d'un type de sollicitation particulière des puissances de l'âme chez le lecteur. Les penseurs médiévaux ont théorisé de manière complexe les rapports de la mémoire, de l'entendement et de la volonté, avec les personnes de la Trinité, mais également entre elles. Il était même admis que théologie spéculative, théologie mystique et théologie symbolique se distinguent les unes des autres notamment en ne s'adressant pas à elles de manière identique. La célèbre lettre de Grégoire le Grand à Serenus, évêque de Marseille, glosée par Thomas d'Aquin et Bonaventure dans leur commentaire respectif sur le Livre des Sentences, a fourni jusqu'au XVII siècle le code fondamental du modus operandi de l'image sur l'âme. Sa triple fonction d'édification des simples, de fixation de l'exemple du Christ et des saints, et d'encouragement à la dévotion,

\footnotetext{
33 «El más grosero de los discípulos de Aristóteles será más fuerte que Aristóteles y Platón, será más elegante orador que Cicerón y Demóstenes. », ibid.

${ }^{34}$ Ralph DEKONINCK, Ad imaginem. Statuts, fonctions et usages de l'image dans la littérature spirituelle jésuite du XVII siècle, op. cit., p. 14-15, expose une conception commune à la plupart des mouvements spirituels et selon laquelle l'image, dans ses fondements théoriques, possède trois modes d'existence - la trace, l'image et la ressemblance - qui correspondent à trois fonctions distinctes - mnémonique, didactique et émotive. Ces associations recoupent, in fine, les trois degrés de l'expérience spirituelle. La trace, avec son rôle mnémonique, relève de la philosophie naturelle et s'avère être le propre de la voie purgative, tandis que dans la voie illuminative, l'image, en tant que représentation mentale, intéresse la théologie symbolique et s'adresse à l'intelligence. Enfin, au stade contemplatif ou mystique, l'esprit tend à la contemplation directe de la vérité au moyen de la ressemblance.
} 
faisait d'elle un instrument de choix pour remédier aux défauts de l'entendement, à la mollesse de la volonté et à la labilité de la mémoire ${ }^{35}$.

Si la finalité suprême et bien souvent implicite dans les traités tels que Leche de la fe del príncipe christiano reste bien le movere, ou plutôt excitare devotionis affectum, il convient de s'interroger sur la nature de cette delectatio recherchée par Maluenda à travers la multiplication des images verbales et discursives. Dans cette triade cicéronienne, c'est la notion la plus suspecte, quoiqu'elle ne soit pas confondue avec la coupable voluptas. À l'instar de spirituels du XVII ${ }^{\mathrm{e}}$ siècle $^{36}$, le franciscain de Burgos ne semble pas attribuer de dimension esthétique à l'image. Du moins n'en fait-il pas état. En effet, la préoccupation pour « refrescar los ojos de los lectores » ne semble pas désigner une expérience esthétique. Les apologues et autres historiettes disséminées dans le traité, qualifiées de "sabrosas y olorosas ", sont d'ailleurs les seuls éléments pouvant expressément soulager la vue d'un lecteur qui parcourt un texte dont le propos général reste austère. Maluenda ne tient donc pas les images verbales pour un élément décoratif à proprement parler. Ce faisant, il nous révèle sa conception utilitariste de l'image prosaïque. Le rôle qu'il lui assigne dépasse de loin la recherche d'une facétie qui ne pointe pas davantage dans les vignettes tirées de la littérature profane.

Pour bien saisir le rôle des images dans l'efficace spirituelle du Tratado llamado leche de la fe del principe christiano, il est nécessaire de tirer toutes les conséquences de leur sélection dans un fonds commun à tout lecteur, sans aucun préjugé face à la noblesse de certaines et à la bassesse d'autres, depuis les œuvres les plus grandioses de la nature jusqu'aux réalités du quotidien domestique. Une grande majorité d'entre elles semblent avoir en partage d'être des «métaphores mortes». En reprenant la formule de Ricœur, nous voulons signifier la double absence d'originalité de ces images. À partir de la distinction entre événement et sens, Ricœur séparait les métaphores vives, existant dans un contexte donné, créations linguistiques pouvant être appelées à "devenir une signification usuelle et [à] s'ajouter à la polysémie des entités lexicales ", des métaphores mortes. Il concluait ainsi sa réflexion: "lorsque l'effet de sens que nous appelons métaphore a rejoint le changement de sens qui augmente la polysémie, la métaphore n'est déjà plus vive, mais morte. ${ }^{37}$ » Il en va ainsi des images verbales du Tratado llamado leche de la fe del principe christiano, qu'elles soient ou non de véritables métaphores. Tout d'abord, elles sont rarement originales et proviennent plus ou moins directement des Écritures ou de l'immense fonds exégétique des Pères et docteurs de l'Église. Ces autorités sont d'ailleurs succinctement référencées dans des annotations marginales fréquentes bien que non systématiques. À ce propos, un passage du prologue assume qu'il faut rendre aux docteurs ce qui est aux docteurs et donc à Maluenda ce qui est à Maluenda, autrement dit bien peu de chose. Dans une assez longue liste sont énumérées puis attribuées méthodiquement à chacun de leurs concepteurs les images verbales par lesquelles les merveilles de Jésus-Christ sont figurées :

Porque las maravillas de Jesucristo relatadas en los sagrados evangelios son los días espirituales, según dize san Agustín. Y son las prendas espirituales que dio el Redemptor a su

\footnotetext{
35 C'est un des passages les plus importants de ce texte : «Elles [les images] furent en effet introduites pour une triple raison, à savoir à cause de l'inculture des simples (propter simplicium ruditatem), à cause de la lenteur des affects (affectuum tarditatem) et à cause de la fragilité de la mémoire (memoria labilitatem). ", cité dans LECERCLE, François, Le signe et la relique. Les théologies de l'image à la Renaissance, thèse de doctorat d'État sous la direction d'Henri Weber, soutenue en septembre 1987 à l'Université Montpellier III-Paul Valéry, p. 34-35.

36 Sur ce point, voir DEKONINCK, Ralph, Ad imaginem. Statuts, fonctions et usages de l'image dans la littérature spirituelle jésuite du XVII' siècle, op. cit., p. 113-127.

${ }^{37}$ RiCceUr, Paul, La métaphore vive (1975), Paris, Seuil, 1997, p. 127.
} 
Iglesia, según dize san Juan Boca de Oro. Y son las vaxillas y vasos de oro y de plata que tiene el aparador de la Iglesia para servicio de los manjares espirituales de las virtudes del Redemptor, según dize san Bernardo. Y son los sellos pontificales para autorizar la verdad y la bula plenaria de la vida santísima de Jesucrito, según dize Juan Gersón. Y son los arneses espirituales con los quales se arman los justos, según dize san Gregorio. Y son las maravillas del Redemptor las granadas maduras y coloradas espirituales que sanan las dolencias del ánima, según dize san Gregorio. Y son las rosas y las flores celestiales que dan olor de divinidad, según dize san Bernardo. Y son las almenas espirituales de oro y de plata y de zafiros y de oro de verdad en el muro de la Iglesia, según dize san Gregorio. Y son los panes regalados para manjar de los hijos y mayorazgos de los reynos de gloria, según dijo el Redemptor a la Cananea, y según declara un dotor de la horden de san Benito. Y son las canales de la lluvia del cielo, según dize san Juan Boca de Oro...38

En dévoilant ses sources, Maluenda ne fait donc que mettre lui-même en pratique ce qu'il louait et recommandait au tout début de l'ouvrage. Il déterre les trésors spirituels enfouis, bien que son humilité l'oblige à se représenter lui-même comme une gargouille sèche dans l'Église. Vaisselle d'or, sceaux pontificaux, harnais, grenades, roses et fleurs, pains, gargouilles, épis de blé, côtoient Augustin, Jean Chrysostome, Bernard, Gerson, Grégoire, Benoît, dans un étonnant capharnaüm d'imagerie spirituelle. On aura remarqué, en second lieu, que le choix d'objets familiers à un ample lectorat ne devait pas requérir d'effort particulier de sa part pour se les représenter mentalement. En outre, le lecteur familier des ouvrages de spiritualité ne faisait que retrouver certaines images rebattues, si bien qu'il ne devait fournir aucun effort pour comprendre la nature du lien métaphorique qui préside à son association. Pour un lecteur plus simple, Maluenda prend soin de toujours l'expliciter, leur infligeant une seconde mort en quelque sorte. En effet, quelle que puisse être l'ampleur du travail d'élucidation du lien entre image et réalité spirituelle ou théologique, tout lecteur s'en trouve systématiquement privé.

La systématisation de l'explicitation, dans le corps du texte, va parfois jusqu'à donner l'impression d'un discours subissant une espèce de dérive métaphorique, comme si la plus ou moins libre association entre les images et les passages de décodage étaient le moteur de la pensée spirituelle. Ainsi, parmi les prolongements donnés à l'image du ver à soie, ou issus de celle-ci, se trouve le mûrier. En réalité, le mûrier se décline en feuilles, qui sont les ornements de l'arbre comme la doctrine christique est celui du chrétien, et en mûres, les plaies du Christ en vertu d'une analogie chromatique. Mais le texte ne s'arrête pas en si bon chemin. À la faveur d'une homonymie, celle de la mûre, mora comme fruit du mûrier, moral, mais également de la ronce, zarza mora, Maluenda juxtapose fruits du Nouveau Testament et de l'Ancien, jusqu'à arriver au buisson-ardent, zarza ardiente, cultivée par le Christjardinier, dans une parfaite image de concordance vétéro et novo-testamentaire, inspirée une fois encore de saint Augustin :

Y como quando un grande ortolano y primero enjeridor enjere (sic) en una grande zarza mora unas púas de un moral de muy dulces frutas no destruye la zarza antes la honra y la pone en perfeción que tenga espinas y que lleve frutas suaves y dulces agenas de espinas. Deste estilo el Redemptor muy grande ortolano y enjertador plantó y enjertó su evangelio sagrado en el tronco de la ley vieja, quitadas las espinas de las cerimonias legales las quales puso encima de su cabeza cumpliéndolas y aprovándolas por buenas antes del tiempo del evangelio y después que se enjertó el evangelio en la ley son las frutas todas unas, y las obras evangélicas y cristianas son muy dulces y suaves como moras dulces del moral, ajenas de las

${ }^{38}$ Maluenda, Luis de, Tratado llamado leche de la fe del principe christiano, op. cit., fol. C iii r-v. 
espinas y de las moras zarzas de las cerimonias legales y de las obras pasadas y secas de la ley vieja... ${ }^{39}$

En dépit des apparences, nous sommes davantage en présence d'une méthode de dérivation métaphorique que d'une réelle dérive, l'auteur épuisant ici toutes les mutations possibles d'une même image. Le franciscain n'est pas exactement en proie à une imagination débordante puisqu'il n'est pas l'auteur de ces images mais plutôt l'artisan de leur rencontre et de leur agencement par l'effet de son art consommé de la compilation. Il recherche bel et bien, en revanche, à neutraliser cette faculté imaginative dans l'âme de son lecteur.

Pour ce faire, il sature sa mémoire d'un visible lui-même au bord de la saturation dans le texte, tant d'un point de vue quantitatif que qualitatif : tout d'abord, par le recours à des images dont la représentation mentale ne requiert aucun effort tant elles sont familières; ensuite, en annulant toute intervention de l'entendement qui n'a pas à dévoiler une correspondance, à démasquer un lien, dans la mesure où le traité se charge de le faire pour lui. Maluenda ne semble rechercher qu'à produire et à entretenir un écho mémoriel qui va faciliter l'affermissement d'une doctrine spirituelle réductible à des entités matérielles visualisables. ${ }^{40}$ Par conséquent, de même que les métaphores sont mortes, voire tuées par Maluenda, la mémoire est dépourvue, dans son fonctionnement, de tout élément dynamique qui pourrait stimuler, dans l'entendement, l'imagination du lecteur. Quand bien même celle du plus vif d'entre eux échapperait au contrôle que Maluenda parait vouloir lui imposer, elle serait rattrapée, dans sa course, par ce discours on ne peut plus prévenant dans sa volonté d'explicitation. À l'inverse, le plus méfiant de ces lecteurs peut également être rassuré, le franciscain dévoile ses sources quand le propos semble trop hardi " porque no piense ninguno que esto es sueño deste pecador $»^{41}$.

Même s'il ne possédait pas son apparente exhaustivité, s'il était de dimension plus modeste ou même s'il avait renoncé à l'exposition d'un système, le Tratado llamado leche de la fe del príncipe christiano mériterait, pour toutes les raisons évoquées, d'être considéré comme une somme. Dans sa farouche volonté de démentir l'adage populaire selon lequel comparaison n'est pas raison, il prétend annuler toute tension herméneutique qui offrirait à son lecteur une échappatoire vers un au-delà de son propre discours, espace clos d'une liberté spirituelle que l'image verbale, telle qu'elle est pensée et maniée, bride. En somme, l'imaginaire de Maluenda, qui devient celui de son lecteur, est réductible à l'imagerie même du traité. Dans son intention totalisante, voire totalitaire, ce liber idiotarum contient même des lieux de récréation pour celui-ci.

Après bien des atermoiements, le 13 décembre 1545, une poignée de pères se réunissaient à Trente pour la session inaugurale d'un Concile longtemps attendu. Moins d'un an auparavant, le 16 janvier de cette même année, Leche de la fe del principe christiano sortait des presses de Juan de Junta, à Burgos. Alors que l'assemblée conciliaire ne donnera sa réponse définitive aux questions soulevées par la Réforme, mais profondément

\footnotetext{
${ }^{39}$ Maluenda, Luis de, Tratado llamado leche de la fe del príncipe christiano, op. cit., fol. A iiii $\mathrm{r}$.

${ }^{40}$ Les images sont mentales, elles ne sont pas disposées selon une topographie et dans un ordre particuliers dans un décor donné, pas plus qu'elles ne sont associées à des numéros : l’intention ne semble pourtant pas éloignée de ce qui soutient le fonctionnement des ars memoria. Il est d'ailleurs assez significatif qu'une liste de Pères et docteurs de l'Église-vers à soie suive immédiatement le déchiffrement de cette métaphore : le lecteur pouvait ainsi, en retenant leur nom, les faire entrer dans cette catégorie.

${ }^{41}$ Maluenda, Luis de, Tratado llamado leche de la fe del principe christiano, op. cit., fol. cclxiii v.
} 
enracinées dans la chrétienté depuis le début du $X V^{\mathrm{e}}$ siècle, que dix-huit ans plus tard, Maluenda formule la sienne propre. Bien entendu, le texte fourmille d'allusions à « este perro de Mahoma » et à ses sectateurs, ainsi qu'à Luther - qui meurt l'année suivante - et aux défenseurs du protestantisme. L'ouvrage sera d'ailleurs partiellement traduit en allemand en 1604 , puis en $1608^{42}$. Il s'agit là de la plus évidente de ses dimensions apologétiques où l'iconophilie, voire l'iconomanie, devient une arme brandie contre la religion iconomaque ${ }^{43}$.

Mais il en possède toutefois une autre. Elle relève d'une question plus profonde, plus sensible, qui s'est déjà posée en Castille, comme en témoigne l'activité inquisitoriale des années 1520-1540. Il s'agit du mouvement illuministe et de ces beatos et beatas qui reviennent fréquemment sous la plume du franciscain et qui ne sont qu'une manifestation supplémentaire d'une hérésie plus générale, au même titre que le luthéranisme. Dans un des nombreux développements de la métaphore de l'eau, les saints et leur doctrine représentent l'eau de roche, source de santé spirituelle.

Deste estilo los libros excelentes que los santos doctores de la Yglesia escrivieron y trabajaron son los pozos y las fuentes de aguas de doctrinas sanas las quales sustentan la sanidad de la fe de la Yglesia y las personas que se crían y gozan de las aguas de las dotrinas de los libros de los santos doctores de escripturas y tratados copilados de las sentencias de los libros de los santos dotores biben sanos en la fe, y libres de sectas y de herejías y de herrores, y tienen bivos colores de caridad y de las virtudes christianas, y tienen las cabeças sanas de los vaguidos y de las piedras de los herrores y engaños de los ereges y de las sectas de luteranos, y dexados y alumbrados, y de bruxos y de beatos, las quales setas y otras semejantes jamás faltarán en la Yglesia hasta el fin del mundo. Según dize san Jerónimo y un dotor teólogo, son las piedras finas las quales quando una vez se crían y se asientan en las cabeças de las personas en especial si son las personas de letras, y si son mujeres muy tarde salen las piedras de las setas de sus cabeças, y los que se crían con las aguas sanas de las dotrinas de los santos tienen los ojos del ánima sanos, y los oídos espirituales limpios...44

Le texte poursuit en opposant à cette eau de source les eaux saumâtres et stagnantes «de las lagunas », des beautés rhétoriques dépourvues des sentences des saints docteurs, à l'instar du contenu des livres de Luther. Bues, elles sont sources de nombreux maux ${ }^{45}$. En somme, ce dont les alumbrados sont coupables aux yeux de Maluenda, c'est de faire d'une liberté exégétique acquise aux dépens de l'exercice du magistère de l'Église la condition d'un incontrôlable et fallacieux épanouissement spirituel. Or l'autorité dogmatique et religieuse de l'Église atteint un degré d'affirmation particulièrement élevé dans le traité de Maluenda, tant dans son principe générateur d'hyper-compilation d'autorités que dans la sollicitation si particulière du monde visible dans son discours, la rencontre de ces deux aspects définissant un cadre d'expérience spirituelle remarquablement étroit, voire étriqué.

Ce faisant, et sans le savoir, Luis de Maluenda, prend en outre position de manière très manifeste - et visionnaire - contre une des principales innovations spirituelles du

\footnotetext{
42 Il s'agit des deux ouvrages suivants : Spiegel eines christlichen Fürsten..., Munich, sans mention d'éditeur, 1604, et Von den grossen Wunderzeichen, Die sowol im Himmel alß auff Erden geschehen seind, und was noch für Zeichen biß an den Jüngsten Tag geschehen werden ..., Munich, Berg vnd Hainrich, 1608.

${ }^{43}$ Les protestants dénonçaient tout spécialement la trop grande humanisation des représentations et la confusion croissante entre signe et chose signifiée. Pour eux, l'image est un écran qui déforme et empêche d'accéder à la création divine. Elle est le produit de l'avilissement dû au péché. Cf. DEKONINCK, Ralph, $A d$ imaginem. Statuts, fonctions et usages de limage dans la littérature spirituelle jésnite du XVII siècle, op. cit., p. 24 et 52.

${ }^{44}$ MALUENDA, Luis de, Tratado llamado leche de la fe del príncipe christiano, op. cit., fol. A vi v.

${ }^{45}$ Ibid.
} 
temps : la composition de lieu des Exercices spirituels d'Ignace de Loyola. Remis sur le métier pendant plus de dix ans, l'opuscule ne connut sa forme définitive qu'en 1544 et ne fut publié que quatre ans plus tard. Il est donc absolument impossible que Maluenda ait eu connaissance du texte fondateur de la spiritualité jésuite. Sans le savoir donc, ou plutôt sans identifier clairement une cible qui pouvait se confondre dans une nébuleuse spirituelle qui plongeait ses racines dans la Devotio moderna, Leche de la fe del principe christiano aiguisait, dans la promotion d'un certain type d'images en matière spirituelle, une arme contre une spiritualité au sein de laquelle la stimulation de l'imagination était appelée à jouer un rôle fondamental. Après bien des déboires, le succès de la Compagnie de Jésus et le rôle de tout premier plan qu'elle était appelée à jouer dans la mise en application du programme de la Contre-Réforme auraient sans doute balayé le franciscain et son œuvre si l'Inquisition ne s'était pas elle-même chargée de les rendre invisibles. 